\title{
Finite Difference Method for Pricing of Indonesian Option under a Mixed Fractional Brownian Motion
}

\author{
Chatarina Enny Murwaningtyas ${ }^{1,2, *}$, Sri Haryatmi Kartiko ${ }^{1}$, Gunardi ${ }^{1}$, \\ Herry Pribawanto Suryawan ${ }^{3}$ \\ ${ }^{1}$ Department of Mathematics, Universitas Gadjah Mada, Yogyakarta, Indonesia \\ ${ }^{2}$ Department of Mathematics Education, Universitas Sanata Dharma, Yogyakarta, Indonesia \\ ${ }^{3}$ Department of Mathematics, Universitas Sanata Dharma, Yogyakarta, Indonesia
}

Received June 8, 2020; Revised August 10, 2020; Accepted August 25, 2020

Cite This Paper in the following Citation Styles

(a): [1] Chatarina Enny Murwaningtyas, Sri Haryatmi Kartiko, Gunardi, Herry Pribawanto Suryawan, "Finite Difference Method for Pricing of Indonesian Option under a Mixed Fractional Brownian Motion," Mathematics and Statistics, Vol. 8, No. 5, pp. 100-109, 2020. DOI: 10.13189/ms.2020.080516.

(b): Chatarina Enny Murwaningtyas, Sri Haryatmi Kartiko, Gunardi, Herry Pribawanto Suryawan , (2020). Finite Difference Method for Pricing of Indonesian Option under a Mixed Fractional Brownian Motion. Mathematics and Statistics, 8(5), 100-109. DOI: 10.13189/ms.2020.080516.

Copyright $(2020$ by authors, all rights reserved. Authors agree that this article remains permanently open access under the terms of the Creative Commons Attribution License 4.0 International License

\begin{abstract}
This paper deals with an Indonesian option pricing using mixed fractional Brownian motion to model the underlying stock price. There have been researched on the Indonesian option pricing by using Brownian motion. Another research states that logarithmic returns of the Jakarta composite index have long-range dependence. Motivated by the fact that there is long-range dependence on logarithmic returns of Indonesian stock prices, we use mixed fractional Brownian motion to model on logarithmic returns of stock prices. The Indonesian option is different from other options in terms of its exercise time. The option can be exercised at maturity or at any time before maturity with profit less than ten percent of the strike price. Also, the option will be exercised automatically if the stock price hits a barrier price. Therefore, the mathematical model is unique, and we apply the method of the partial differential equation to study it. An implicit finite difference scheme has been developed to solve the partial differential equation that is used to obtain Indonesian option prices. We study the stability and convergence of the implicit finite difference scheme. We also present several examples of numerical solutions. Based on theoretical analysis and the numerical solutions, the scheme proposed in this paper is efficient and reliable.
\end{abstract}

Keywords Indonesian Option Pricing, Mixed Fractional Brownian Motion, Finite Difference

\section{Introduction}

The Jakarta Stock Exchange, currently called the Indonesia Stock Exchange after merging with the Surabaya Stock Exchange, launched an option on October 6, 2004. The option traded in Indonesia is different to the usual options. An Indonesia option [1] is an American option that is given a barrier, but the Indonesian option only has maximum gain of $10 \%$ of a strike price. The option price depends on the weighted moving average (WMA) price of the underlying stock price. The WMA price is a ratio of the total value of all transactions to the total volume of the stock traded in the last 30 minutes. Calculating the Indonesia option by using the WMA price is not easy due to model complexity. If the WMA price is calculated during the last 30 minutes, then the WMA price and the stock price do not differ in terms of value. This study assumed the WMA price is equal to the stock price.

In Indonesian options, if a stock price hits the barrier value, then the option will be exercised automatically with a gain of $10 \%$ of a strike price. On the contrary, if the stock price does not hit the barrier, then the option can be exercised any time before or at the maturity date. When the stock price does not hit the barrier, option buyers tend to wait until maturity. This is due to the fact that the barrier value is close enough to the strike price and the maximum duration of the contract is only 3 months. Therefore, we are interested in studying the pricing of Indonesian options that can be exercised at maturity or when the stock prices hit the barrier.

Gunardi et al. [2] introduced pricing of Indonesian options. The pricing of Indonesian options in [2, 3, 4] used Black- 
Scholes and variance gamma models. The Black-Scholes model used geometric Brownian motion to model logarithmic returns of stock prices. This model assumes that logarithmic returns of stock prices ware normally and independent identically distributed (iid). However, empirical studies have shown that logarithmic returns of stock prices usually exhibit properties of self-similarity, heavy tails, and long-range dependence [5, 6, 7]. Even Cajueiro [5] and Fakhriyana [7] stated that returns of the Jakarta Composite Index have long-range dependence properties. In this situation, it is suitable to model the stock price using a fractional Brownian motion (FBM).

To use a FBM in option pricing, we must define a riskneutral measure and the Itô formula, with analog in Brownian motion. $\mathrm{Hu}$ and $\varnothing \mathrm{ksendal}$ [8] contributed to finding the Itô formula that can be used in the FBM model. However, the determination of option prices still had an arbitrage opportunity. Cheridito [9] proposed a mixed fractional Brownian motion (MFBM) to reduce an arbitrage opportunity. In this paper, we employ the MFBM on the Indonesian option pricing to reduce the arbitrage opportunity.

In the stock market, there are many types of options traded. European and American options are standard or vanilla options. European options can be exercised at maturity, whereas American options can be exercised at any time during the contract. Pricing of European options using MFBM has been studied in $[10,11]$. Chen et al. [12] investigated numerically pricing of American options under the generalization of MFBM. Options that have more complicated rules than vanilla options are called exotic options. Examples of exotic options are Asian options, rainbow options, currency options, barrier options, and also Indonesian options. Rao [13] and Zang et al. [14] discussed the pricing of Asian power options under MFBM. Wang [15] explored the pricing of Asian rainbow options under FBM. Currency options pricing under FBM and MFBM has been studied in $[16,17,18]$. Numerical solution of barrier options pricing under MFBM have been evaluated by Ballestra et al. [19].

Indonesian option is one type of barrier options. Because analytic solutions for barrier options are not easy to find [19], we determine Indonesian options using numerical solutions. One numerical solution that can be used is the finite difference method discussed in [20]. The purpose of this paper is to determine Indonesian option prices under the MFBM model using the finite difference method. In this article, we also show that the resulting finite difference scheme is stable and convergent.

\section{An option pricing model by using MFBM}

A mixed fractional Black Scholes market is a model consisting of two assets, one riskless asset (bank account) and one risky asset (stock). A bank account satisfies

$$
d A_{t}=r A_{t} d t, \quad A_{0}=1
$$

where $A_{t}$ denotes a bank account at time $t, t \in[0, T]$, with an interest rate $r$. Meanwhile, a stock price is modeled by using an MFBM defined in Definition A.2 (Appendix A). The stock price satisfies

$$
d S_{t}=\mu S_{t} d t+\alpha \sigma S_{t} d \hat{B}_{t}+\beta \sigma S_{t} d \hat{B}_{t}^{H}, \quad S_{0}>0,
$$

where $S_{t}$ denotes a stock price at time $t, t \in[0, T]$, with an expected return $\mu$ and a volatility $\sigma, \hat{B}_{t}$ is a Brownian motion, $\hat{B}_{t}^{H}$ is an independent FBM of Hurst index $H$ with respect to a probability measure $\hat{\mathbb{P}}^{H}$.

According to the fractional Girsanov theorem [21], it is known that there is a risk-neutral measure $\mathbb{P}^{H}$, so that if $\alpha \sigma \hat{B}_{t}+\beta \sigma \hat{B}_{t}^{H}=\alpha \sigma B_{t}+\beta \sigma B_{t}^{H}-\mu+r$ is

$$
d S_{t}=r S_{t} d t+\alpha \sigma S_{t} d B_{t}+\beta \sigma S_{t} d B_{t}^{H}, \quad S_{0}>0 .
$$

Lemma 1. The stochastic differential equation (1) admits a solution

$$
S_{t}=S_{0} \exp \left(r t-\frac{1}{2}(\alpha \sigma)^{2} t-\frac{1}{2}(\beta \sigma)^{2} t^{2 H}+\alpha \sigma B_{t}+\beta \sigma B_{t}^{H}\right) .
$$

In mathematical finance, the Black-Scholes equation is a partial differential equation (PDE) which is used to determine the price of an option based on the Black-Scholes model. The Black-Scholes type differential equation based on an MFBM is constructed in the following theorem.

Theorem 2. Let $V(t, S)$ be an option value that depends on a time $t$ and a stock price $S$. Then, under an MFBM model, $V(t, S)$ satisfies

$$
\begin{aligned}
\frac{\partial V}{\partial t} & +r S \frac{\partial V}{\partial S}+\frac{1}{2}(\alpha \sigma S)^{2} \frac{\partial^{2} V}{\partial S^{2}} \\
& +(\beta \sigma S)^{2} H t^{2 H-1} \frac{\partial^{2} V}{\partial S^{2}}-r V=0 .
\end{aligned}
$$

\section{A Finite Difference Method for In- donesian option pricing}

An Indonesian option is an option that can be exercised at maturity or at any time before maturity but the profit does not exceed 10 percent of the strike price. The option will be exercised automatically if the stock price hits a barrier price. The barrier price in an Indonesian option is $110 \%$ of the strike price for a call option and $90 \%$ of the strike price for a put option. Because the benefits of an Indonesian option is very small, more option contract holders often choose to exercise their contracts at maturity. In other words, an Indonesian option is an option that can be exercised at maturity or when the stock hits the barrier price.

Let $L$ is a barrier of an Indonesian option and $t_{L}$ is the first time of the stock price hitting the barrier;

$$
t_{L}=\min \left\{t \mid t \in[0, T], S_{t} \geq L\right\} .
$$

An Indonesian call option with a strike price $K$ can be exercised at maturity $T$ or until the stock price of $S_{t}$ hits the barrier at $L=1.1 K$. The payoff function at time $T$ of the call option can be expressed as follows :

$$
f\left(S_{T}\right)= \begin{cases}S_{T}-K & \text { if } t_{L}>T \\ (L-K) e^{r\left(T-t_{L}\right)} & \text { if } t_{L} \leq T\end{cases}
$$


Similarly, the payoff function at time $T$ of an Indonesian put option with barrier price $L=0.9 \mathrm{~K}$ can be expressed as follows :

$$
f\left(S_{T}\right)= \begin{cases}K-S_{T} & \text { if } t_{L}>T \\ (K-L) e^{r\left(T-t_{L}\right)} & \text { if } t_{L} \leq T\end{cases}
$$

The partial differential equation used in the Indonesian option pricing is a PDE with a final time condition. Because finite difference methods usually use an initial time condition, we make changes on variable $\tau$ i.e. $\tau=T-t$. Under this transformation, PDE (3) becomes,

$$
\begin{aligned}
\frac{\partial V}{\partial \tau} & -r S \frac{\partial V}{\partial S}-\frac{1}{2}(\alpha \sigma S)^{2} \frac{\partial^{2} V}{\partial S^{2}} \\
& -(\beta \sigma S)^{2} H(T-\tau)^{2 H-1} \frac{\partial^{2} V}{\partial S^{2}}+r V=0
\end{aligned}
$$

We must set up a discrete grid in this case with respect to stock prices and time to solve the PDE by finite difference methods. Suppose $S_{\max }$ is a suitably large stock price and in this case $S_{\max }=L$. We need $S_{\max }$ since the domain for the PDE is unbounded with respect to stock prices, but we must bound it in some ways for computing purposes. The grid consists of points $\left(\tau_{k}, S_{j}\right)$ such that $S_{j}=j \Delta S$ and $\tau_{k}=k \Delta \tau$ with $j=0,1, \ldots, M$ and $k=0,1, \ldots, N$.

Using Taylor series expansion, we have

$$
\begin{gathered}
\frac{V_{j}^{k}-V_{j}^{k-1}}{\Delta \tau}=\frac{\partial V}{\partial \tau}+\mathcal{O}(\Delta \tau), \\
\frac{V_{j+1}^{k}-V_{j-1}^{k}}{2 \Delta S}=\frac{\partial V}{\partial S}+\mathcal{O}\left((\Delta S)^{2}\right),
\end{gathered}
$$

and

$$
\frac{V_{j+1}^{k}-2 V_{j}^{k}+V_{j-1}^{k}}{(\Delta S)^{2}}=\frac{\partial^{2} V}{\partial S^{2}}+\mathcal{O}\left((\Delta S)^{2}\right) .
$$

Substitution of (8), (9) and (10) in (7) yields

$$
\begin{aligned}
\frac{V_{j}^{k}-V_{j}^{k-1}}{\Delta \tau} & -r j \Delta S \frac{V_{j+1}^{k}-V_{j-1}^{k}}{2 \Delta S}-\frac{(\alpha \sigma)^{2}}{2}(j \Delta S)^{2} \frac{V_{j+1}^{k}-2 V_{j}^{k}+V_{j-1}^{k}}{(\Delta S)^{2}} \\
& -(\beta \sigma)^{2}(j \Delta S)^{2} H(T-k \Delta \tau)^{2 H-1} \frac{V_{j+1}^{k}-2 V_{j}^{k}+V_{j-1}^{k}}{(\Delta S)^{2}} \\
& +r V_{j}^{k}=0
\end{aligned}
$$

where the local truncation error is $\mathcal{O}\left(\Delta \tau+(\Delta S)^{2}\right)$. Rewriting (11), we get an implicit scheme as follows

$$
V_{j}^{k-1}=a_{j} V_{j-1}^{k}+b_{j} V_{j}^{k}+c_{j} V_{j+1}^{k},
$$

where

$$
a_{j}=\left(-\frac{1}{2}(\alpha \sigma j)^{2}-(\beta \sigma j)^{2} H(T-k \Delta \tau)^{2 H-1}+\frac{1}{2} r j\right) \Delta \tau,
$$

$b_{j}=\left(1+\left((\alpha \sigma j)^{2}+2(\beta \sigma j)^{2} H(T-k \Delta \tau)^{2 H-1}+r\right) \Delta \tau\right)$, $c_{j}=\left(-\frac{1}{2}(\alpha \sigma j)^{2}-(\beta \sigma j)^{2} H(T-k \Delta \tau)^{2 H-1}-\frac{1}{2} r j,\right) \Delta \tau$.

Using (4) and (5), we can write an initial condition of the Indonesian call option as follows:

$$
V_{j}^{0}= \begin{cases}j \Delta S-K & \text { if } L>j \Delta S, \\ L-K & \text { if } L \leq j \Delta S,\end{cases}
$$

and boundary conditions of the call option as follows:

$$
V_{0}^{k}=0 \quad \text { and } \quad V_{M}^{k}=(L-K) e^{-r k \Delta \tau} .
$$

In another case, using (4) and (6), we get an initial condition and boundary conditions of the Indonesian put option shown below respectively:

$$
V_{j}^{0}= \begin{cases}K-j \Delta S & \text { if } L<j \Delta S, \\ K-L & \text { if } L \geq j \Delta S\end{cases}
$$

and

$$
V_{0}^{k}=0 \quad \text { and } \quad V_{M}^{k}=(K-L) e^{-r k \Delta \tau} .
$$

We analyze the stability and convergence of the implicit finite difference scheme using Fourier analysis. Firstly, we discuss the stability of the implicit finite difference scheme. Let $V_{j}^{k}$ be difference solution of (12) and $U_{j}^{k}$ be another approximate solution of (12), we define a roundoff error $\varepsilon_{j}^{k}=$ $V_{j}^{k}-U_{j}^{k}$. Next, we obtain a following roundoff error equation

$$
\varepsilon_{j}^{k-1}=a_{j} \varepsilon_{j-1}^{k}+b_{j} \varepsilon_{j}^{k}+c_{j} \varepsilon_{j+1}^{k} .
$$

Furthermore, we define a grid function as follows:

$$
\varepsilon^{k}(S)= \begin{cases}\varepsilon_{j}^{k} & \text { if } S_{j}-\frac{\Delta S}{2}<S \leq S_{j}+\frac{\Delta S}{2}, j=1, \ldots, M-1, \\ 0 & \text { if } 0 \leq S \leq \frac{\Delta S}{2} \text { or } S_{\max }-\frac{\Delta S}{2}<S \leq S_{\max }\end{cases}
$$

The grid function can be expanded in a Fourier series below:

$$
\varepsilon^{k}(S)=\sum_{l=-\infty}^{\infty} \xi^{k}(l) \exp \left(\frac{i 2 \pi l S}{S_{\max }}\right), \quad k=1,2, \ldots, N,
$$

where

$$
\xi^{k}(l)=\frac{1}{S_{\max }} \int_{0}^{S_{\max }} \varepsilon^{k}(S) \exp \left(\frac{-i 2 \pi l S}{S_{\max }}\right) d S .
$$

Moreover, we let

$$
\varepsilon^{k}=\left[\varepsilon_{1}^{k}, \varepsilon_{2}^{k}, \ldots, \varepsilon_{N-1}^{k}\right]^{T} .
$$

And we introduce a norm,

$$
\left\|\varepsilon^{k}\right\|_{2}=\left(\sum_{j=1}^{M-1}\left|\varepsilon_{j}^{k}\right|^{2} \Delta S\right)^{\frac{1}{2}}=\left(\int_{0}^{S_{\max }}\left|\varepsilon^{k}(S)\right|^{2} d S\right)^{\frac{1}{2}} .
$$

Further, by using Parseval equality,

$$
\int_{0}^{S_{\max }}\left|\varepsilon^{k}(S)\right|^{2} d S=\sum_{l=-\infty}^{\infty}\left|\xi^{k}(l)\right|^{2},
$$


we obtain

$$
\left\|\varepsilon^{k}\right\|_{2}^{2}=\sum_{l=-\infty}^{\infty}\left|\xi^{k}(l)\right|^{2}
$$

At the moment, we assume that the solution of equation (18) has the following form

$$
\varepsilon_{j}^{k}=\xi^{k} e^{i \omega j \Delta S}
$$

where $\omega=\frac{2 \pi l}{S_{\max }}$ and $i=\sqrt{-1}$. Substituting (19) into (18), we obtain

$$
\begin{aligned}
\xi^{k-1} e^{i \omega j \Delta S} & =a_{j} \xi^{k} e^{i \omega(j-1) \Delta S}+b_{j} \xi^{k} e^{i \omega j \Delta S}+c_{j} \xi^{k} e^{i \omega(j+1) \Delta S} \\
& =\xi^{k} e^{i \omega j \Delta S}\left(a_{j} e^{-i \omega \Delta S}+b_{j}+c_{j} e^{i \omega \Delta S}\right)
\end{aligned}
$$

Equation (20) can be rewritten as follows,

$$
\begin{aligned}
& \xi^{k-1}=\xi^{k}\left(a_{j} e^{-i \omega \Delta S}+b_{j}+c_{j} e^{i \omega \Delta S}\right), \\
& \xi^{k-1}=\xi^{k} \vartheta_{j},
\end{aligned}
$$

where

$$
\vartheta_{j}=a_{j} e^{-i \omega \Delta S}+b_{j}+c_{j} e^{i \omega \Delta S} .
$$

By substituting (13), (14) and (15) into (23), we obtain

$$
\begin{aligned}
\vartheta_{j}= & \left(-(\alpha \sigma j)^{2}-2(\beta \sigma j)^{2} H(T-k \Delta \tau)^{2 H-1}\right) \Delta \tau \cos (\omega \Delta S) \\
& +\left((\alpha \sigma j)^{2}+2(\beta \sigma j)^{2} H(T-k \Delta \tau)^{2 H-1}+r\right) \Delta \tau \\
& -r j i \Delta \tau \sin (\omega \Delta S)+1 .
\end{aligned}
$$

Proposition 3. If $\xi^{k}, k \in \mathbb{N}$, is a solution of (21), then $\left|\xi^{k}\right| \leq$ $\left|\xi^{0}\right|$.

Hence by (19) and Proposition 3, we have the following theorem.

Theorem 4. The difference scheme (12) is unconditionally stable.

Now we analyze the convergence of implicit finite difference scheme. Let $V\left(\tau_{k}, S_{j}\right)$ is exact solution of (7) at a point $\left(\tau_{k}, S_{j}\right)$ and

$$
\begin{aligned}
R_{j}^{k}= & \frac{V\left(\tau_{k}, S_{j}\right)-V\left(\tau_{k-1}, S_{j}\right)}{\Delta \tau}-r j \Delta S \frac{V\left(\tau_{k}, S_{j+1}\right)-V\left(\tau_{k}, S_{j-1}\right)}{2 \Delta S} \\
- & \frac{1}{2}(\alpha \sigma)^{2}(j \Delta S)^{2} \frac{V\left(\tau_{k}, S_{j+1}\right)-2 V\left(\tau_{k}, S_{j}\right)+V\left(\tau_{k}, S_{j-1}\right)}{(\Delta S)^{2}} \\
- & \left((\beta \sigma)^{2}(j \Delta S)^{2} H(T-k \Delta \tau)^{2 H-1}\right) \\
& \quad \times \frac{V\left(\tau_{k}, S_{j+1}\right)-2 V\left(\tau_{k}, S_{j}\right)+V\left(\tau_{k}, S_{j-1}\right)}{(\Delta S)^{2}} \\
& +r V\left(\tau_{k}, S_{j}\right),
\end{aligned}
$$

where $k=1,2, \ldots, N$ and $j=1,2, \ldots, M-1$. Consequently, there is a positive constant $C_{1}^{k, j}$, so as

$$
\left|R_{j}^{k}\right| \leq C_{1}^{k, j}\left(\Delta \tau+(\Delta S)^{2}\right),
$$

then, we have

$$
\left|R_{j}^{k}\right| \leq C_{1}\left(\Delta \tau+(\Delta S)^{2}\right)
$$

where

$$
C_{1}=\max \left\{C_{1}^{k, j} \mid k=1,2, \ldots, N ; j=1,2, \ldots, M-1\right\} .
$$

From (12), (13), (14), (15) and definition $R_{j}^{k}$ in (25), we have

$$
\begin{aligned}
V\left(\tau_{k-1}, S_{j}\right)= & a_{j} V\left(\tau_{k}, S_{j-1}\right)+b_{j} V\left(\tau_{k}, S_{j}\right) \\
& +c_{j} V\left(\tau_{k}, S_{j+1}\right)-\Delta \tau R_{j}^{k} .
\end{aligned}
$$

By subtracting (12) from (27), we obtain

$$
\epsilon_{j}^{k-1}=a_{j} \epsilon_{j-1}^{k}+b_{j} \epsilon_{j}^{k}+c_{j} \epsilon_{j+1}^{k}-\Delta \tau R_{j}^{k},
$$

where an error $\epsilon_{j}^{k}=V\left(\tau_{k}, S_{j}\right)-V_{j}^{k}$. The error equation satisfies a boundary conditions,

$$
\epsilon_{0}^{k}=\epsilon_{M}^{k}=0, \quad k=1,2, \ldots, N,
$$

and an initial condition,

$$
\epsilon_{j}^{0}=0, \quad j=1,2, \ldots, M
$$

Next, we define the following grid functions,

$\epsilon^{k}(S)= \begin{cases}\epsilon_{j}^{k} & \text { if } S_{j}-\frac{\Delta S}{2}<S \leq S_{j}+\frac{\Delta S}{2}, j=1, \ldots, M-1 \\ 0 & \text { if } 0 \leq S \leq \frac{\Delta S}{2} \text { or } S_{\max }-\frac{\Delta S}{2}<S \leq S_{\max }\end{cases}$

and

$R^{k}(S)= \begin{cases}R_{j}^{k} & \text { if } S_{j}-\frac{\Delta S}{2}<S \leq S_{j}+\frac{\Delta S}{2}, j=1, \ldots, M-1, \\ 0 & \text { if } 0 \leq S \leq \frac{\Delta S}{2} \text { or } S_{\max }-\frac{\Delta S}{2}<S \leq S_{\max }\end{cases}$

The grid functions can be expanded in a Fourier series respectively as follows

$$
\epsilon^{k}(S)=\sum_{l=-\infty}^{\infty} \varrho^{k}(l) \exp \left(\frac{i 2 \pi l S}{S_{\max }}\right), \quad k=1,2, \ldots, N,
$$

and

$$
R^{k}(S)=\sum_{l=-\infty}^{\infty} \rho^{k}(l) \exp \left(\frac{i 2 \pi l S}{S_{\max }}\right), \quad k=1,2, \ldots, N
$$

where

$$
\varrho^{k}(l)=\frac{1}{S_{\max }} \int_{0}^{S_{\max }} \epsilon^{k}(S) \exp \left(\frac{-i 2 \pi l S}{S_{\max }}\right) d S
$$

and

$$
\rho^{k}(l)=\frac{1}{S_{\max }} \int_{0}^{S_{\max }} R^{k}(S) \exp \left(\frac{-i 2 \pi l S}{S_{\max }}\right) d S .
$$

Thus, we let

$$
\boldsymbol{\epsilon}^{k}=\left[\epsilon_{1}^{k}, \epsilon_{2}^{k}, \ldots, \epsilon_{N-1}^{k}\right]^{T}
$$

and

$$
\boldsymbol{R}^{k}=\left[R_{1}^{k}, R_{2}^{k}, \ldots, R_{N-1}^{k}\right]^{T},
$$

and we define their corresponding norms

$$
\left\|\epsilon^{k}\right\|_{2}=\left(\sum_{j=1}^{M-1}\left|\epsilon_{j}^{k}\right|^{2} \Delta S\right)^{\frac{1}{2}}=\left(\int_{0}^{S_{\max }}\left|\epsilon^{k}(S)\right|^{2} d S\right)^{\frac{1}{2}},
$$


and

$$
\left\|\boldsymbol{R}^{k}\right\|_{2}=\left(\sum_{j=1}^{M-1}\left|R_{j}^{k}\right|^{2} \Delta S\right)^{\frac{1}{2}}=\left(\int_{0}^{S_{\max }}\left|R^{k}(S)\right|^{2} d S\right)^{\frac{1}{2}}
$$

respectively. By using Parseval equality, we get

$$
\int_{0}^{S_{\max }}\left|\epsilon^{k}(S)\right|^{2} d S=\sum_{l=-\infty}^{\infty}\left|\varrho^{k}(l)\right|^{2}
$$

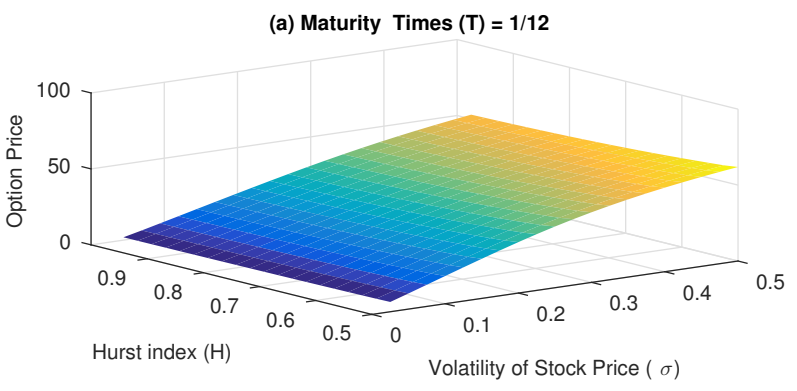

and

$$
\int_{0}^{S_{\max }}\left|R^{k}(S)\right|^{2} d S=\sum_{l=-\infty}^{\infty}\left|\rho^{k}(l)\right|^{2},
$$

respectively. As a consequence, we can show that

$$
\left\|\epsilon^{k}\right\|_{2}^{2}=\sum_{l=-\infty}^{\infty}\left|\varrho^{k}(l)\right|^{2}
$$

and

$$
\left\|\boldsymbol{R}^{k}\right\|_{2}^{2}=\sum_{l=-\infty}^{\infty}\left|\rho^{k}(l)\right|^{2}
$$

Further, we assume that the solution of (28) has the following form

$$
\epsilon_{j}^{k}=\varrho^{k} e^{i \omega j \Delta S}
$$

and

$$
R_{j}^{k}=\rho^{k} e^{i \omega j \Delta S} .
$$

Substituting (33) and (34) into (28), we obtain

$\varrho^{k-1} e^{i \omega j \Delta S}=e^{i \omega j \Delta S}\left(\varrho^{k}\left(a_{j} e^{-i \omega \Delta S}+b_{j}+c_{j} e^{i \omega \Delta S}\right)-\Delta \tau \rho^{k}\right)$.

Equation (35) can be simply rewritten as follows

$$
\varrho^{k-1}=\varrho^{k}\left(a_{j} e^{-i \omega \Delta S}+b_{j}+c_{j} e^{i \omega \Delta S}\right)-\Delta \tau \rho^{k} .
$$

By using equations (13), (14), (15) and (36), we obtain

$$
\begin{aligned}
\varrho^{k-1}= & {\left[\left(-(\alpha \sigma j)^{2}-2(\beta \sigma j)^{2} H(T-k \Delta \tau)^{2 H-1}\right) \Delta \tau \cos (\omega \Delta S)\right.} \\
& +\left((\alpha \sigma j)^{2}+2(\beta \sigma j)^{2} H(T-k \Delta \tau)^{2 H-1}+r\right) \Delta \tau \\
& -r j i \Delta \tau \sin (\omega \Delta S)+1] \varrho^{k}-\Delta \tau \rho^{k} .
\end{aligned}
$$

Equation (37) can be effectively expressed as follows

$$
\varrho^{k}=\frac{1}{\vartheta_{j}} \varrho^{k-1}+\frac{1}{\vartheta_{j}} \Delta \tau \rho^{k},
$$

where $\vartheta_{j}$ is defined in (24).

Proposition 5. Assuming that $\varrho^{k}(k=1,2, \ldots, N)$ is a solution of (37), then there exist a positive constant $C_{2}$, so that

$$
\left|\varrho^{k}\right| \leq C_{2} k \Delta \tau\left|\rho^{1}\right|
$$

The following theorem gives convergence of the different scheme (12).

Theorem 6. The difference scheme (12) is $L_{2}$-convergent, and the convergence order is $\mathcal{O}\left(\Delta \tau+(\Delta S)^{2}\right)$.

(b) Maturity Times $(T)=2 / 12$

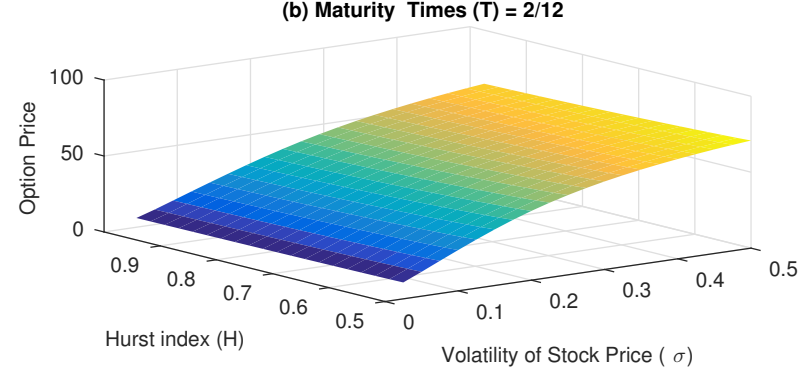

(c) Maturity Times $(\mathrm{T})=3 / 12$

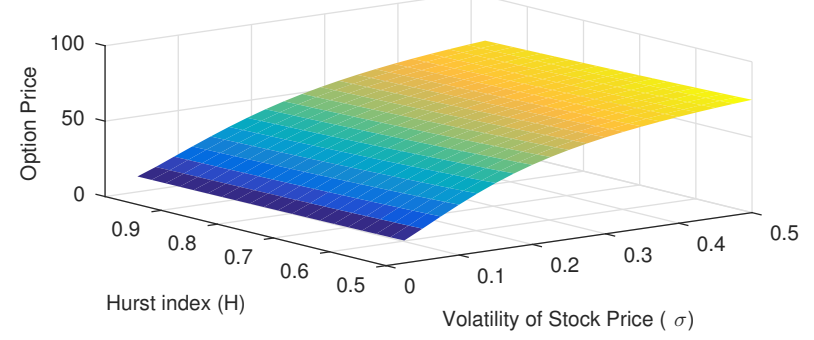

Figure 1. Indonesian option prices on $H$ and $\sigma$ for values $T=\frac{1}{12}, T=\frac{2}{12}$ and $T=\frac{3}{12}$.

\section{Numerical examples and discussions}

An Indonesian option pricing based on an MFBM has been studied. An implicit difference scheme of (7) is given in (12) and initial and boundary conditions of an Indonesian call option is given in (16) and (17), respectively. We provide several numerical results that illustrate the stability and convergence of the finite difference method in calculating an Indonesian call option price using Matlab in this section. In Examples 1, 2 and 3 , we show that the scheme is stable. We also show that the scheme is convergent in Example 4. Furthermore, Example 5 compares the option price generated by the scheme with the exact solution in [2] when $\alpha=0, \beta=1, H=\frac{1}{2}$.

Example 1. An Indonesian call option pricing model is based on (12) where $\alpha=\beta=1$, an initial condition (16) and boundary conditions (17) under the following parameters,

$$
\Delta S=1, \Delta \tau=0.0001, r=0.05, S_{0}=1000, K=1000,
$$

and various values of parameters,

$$
H \in(0.5,1), \sigma \in(0,0.5), T \in\left\{\frac{1}{12}, \frac{2}{12}, \frac{3}{12}\right\}
$$

Figure 1 exhibits the price surface of an Indonesian call option with a change of the Hurst index $(H)$ and a change of 
(a) Volatility of Stock Price $(\sigma)=0.01$

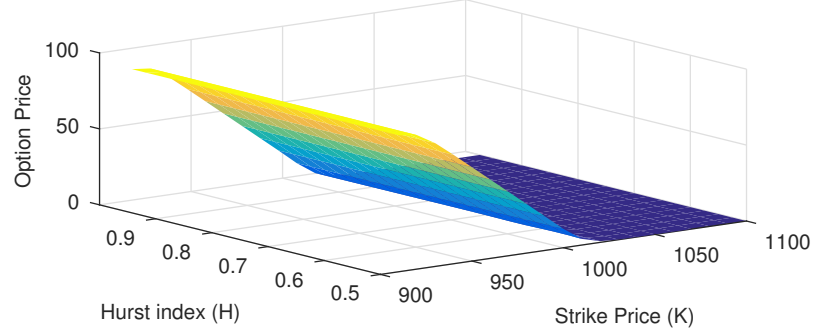

(b) Volatility of Stock Price $(\sigma)=0.05$

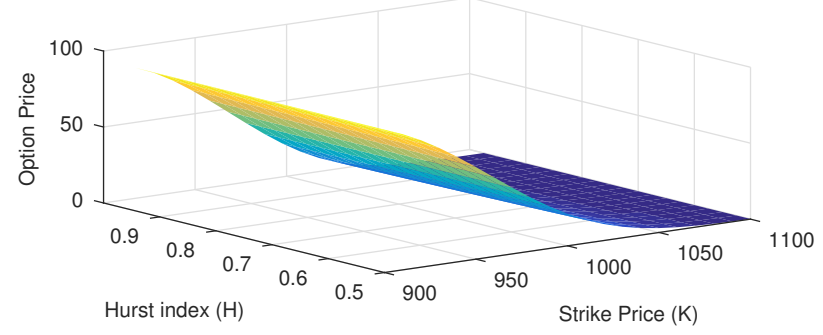

(c) Volatility of Stock Price $(\sigma)=0.1$

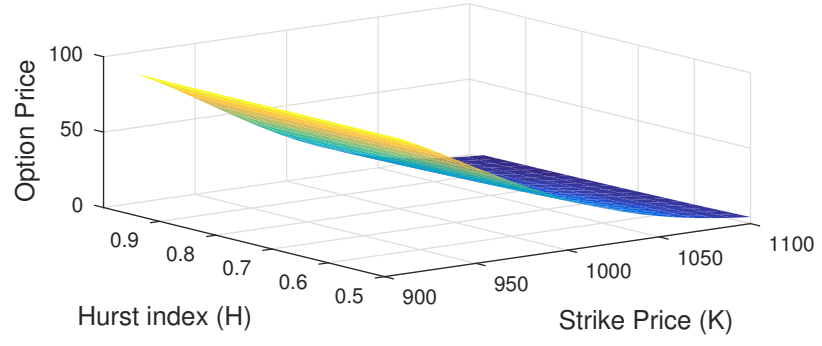

Figure 2. Indonesian option prices on $H$ and $K$ for values $\sigma=0.01, \sigma=$ 0.05 and $\sigma=0.1$.

stock price volatility $(\sigma)$ for difference maturity time $(T)$. The Hurst Index, stock price volatility and maturity time affect option prices. As the Hurst index decreases and the stock price volatility and maturity time increase, we see that the price of Indonesian options increase.

Example 2. Consider an Indonesian call option pricing at (12), (16) and (17) with $\alpha=\beta=1$ and parameters,

$$
\Delta S=1, \Delta \tau=0.0001, r=0.05, S_{0}=1000, T=\frac{3}{12},
$$

and various values of parameters,

$$
H \in(0.5,1), K \in(900,1100), \sigma \in\{0.01,0.05,0.1\}
$$

Figure 2 shows the price surface of an Indonesian call option with a change of Hurst index $(H)$ and a change of strike price $(K)$ for various volatility values of the stock price $(\sigma)$. As the stock price volatility increases, the Hurst index and strike price decrease, we see that the price of Indonesian options increase.

Example 3. Consider an Indonesian call option pricing problem (12), (16) and (17) with $\alpha=\beta=1$ and parameters,

$$
\Delta S=1, \Delta t=0.0001, r=0.05, S_{0}=1000, K=1000,
$$

(a) Volatility of Stock Price $(\sigma)=0.01$

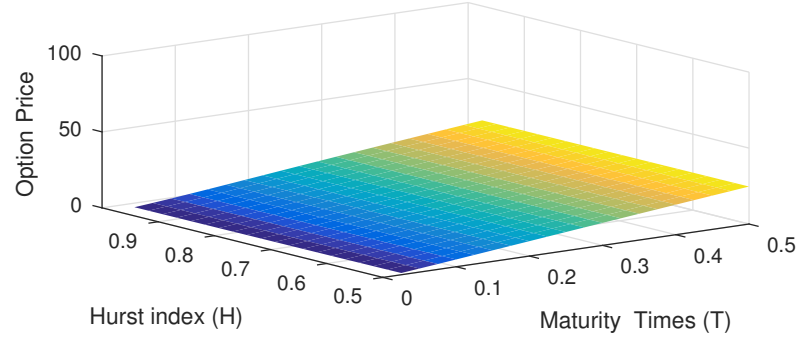

(b) Volatility of Stock Price $(\sigma)=0.05$

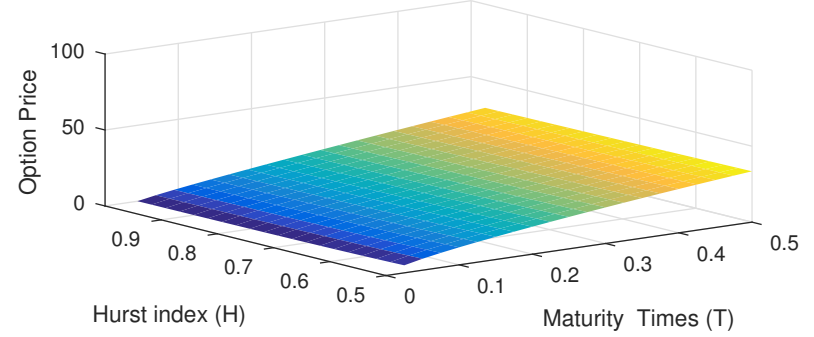

(c) Volatility of Stock Price $(\sigma)=0.1$

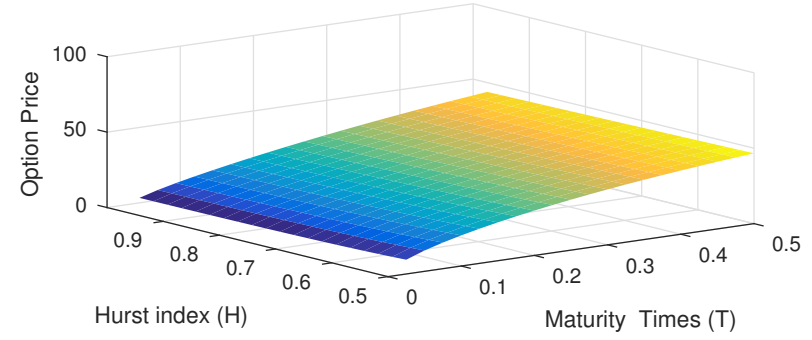

Figure 3. Indonesian option prices with $H$ and $T$ for values of $\sigma=0.01$, $\sigma=0.05$ and $\sigma=0.1$

and various values of parameters

$$
H \in(0.5,1), T \in(0,0.5), \sigma \in\{0.01,0.05,0.1\}
$$

Figure 3 shows the price surface of an Indonesian call option with a change of the Hurst index $(H)$ and a change of maturity time $(T)$ for various values of stock price volatility $(\sigma)$. Similar to the result obtained in Example 1, we see that the price of Indonesian options increase when the stock price volatility and maturity time increase while the Hurst index decreases.

Example 4. Consider an Indonesian call option pricing at (12), (16) and (17) with $\alpha=\beta=1$ and parameters,

$$
r=0.05, \sigma=0.1, T=0.25, S_{0}=1000, K=1000, H=0.7 \text {. }
$$

This example will show the convergence of the scheme (12). The convergence is demonstrated by the difference between consecutive approximation processes in Table 1 . The numerical results from Table 1 confirm the results of the theoretical analysis (B.8) in Theorem 6.

Example 5. Let Indonesian call option pricing at (12), (16) 

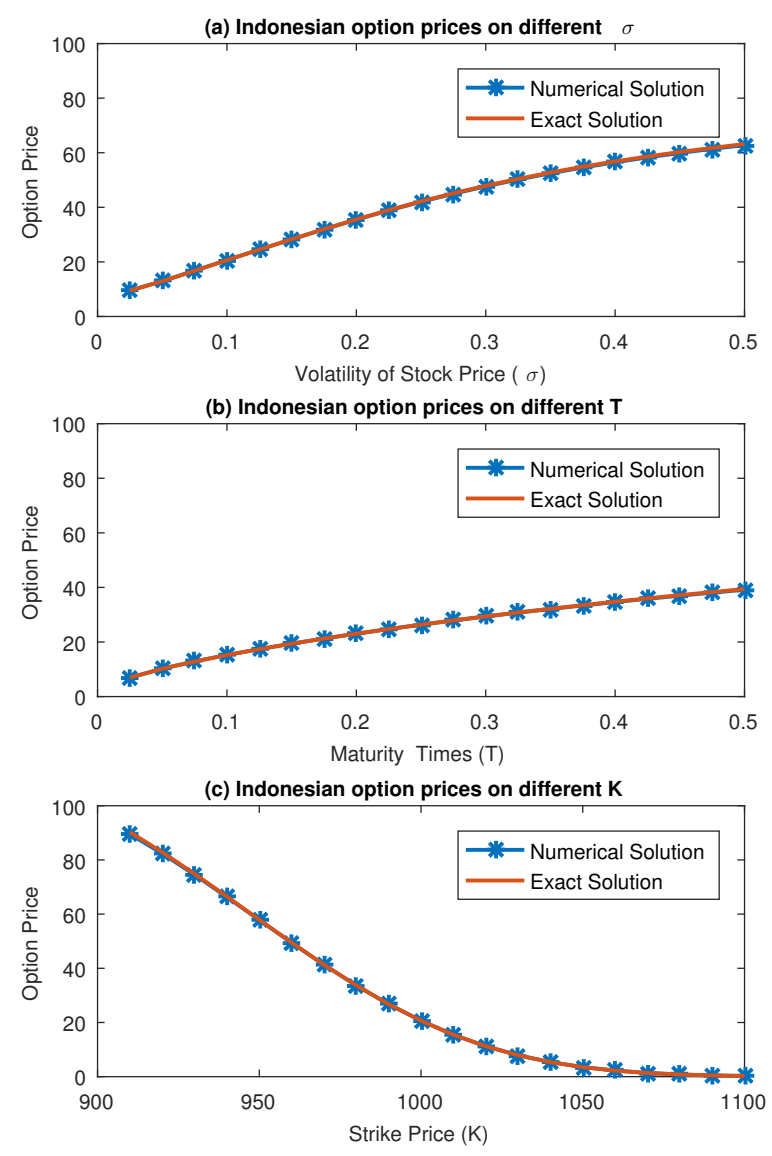

Figure 4. The price of Indonesian options uses the exact and numerical solution for $H=\frac{1}{2}$.

and (17) with $\alpha=0, \beta=1, H=\frac{1}{2}$ and parameters,

$$
\begin{gathered}
\Delta S=1, \Delta \tau=0.0001, r=0.05, \sigma=0.1, T=\frac{2}{12}, \\
S_{0}=1000, K=1000 .
\end{gathered}
$$

Equation (7) with $\alpha=0, \beta=1$ and $H=\frac{1}{2}$ is a stock price model under a Brownian motion. Figure 4 shows the comparison of numerical and exact solutions of Indonesian option prices for stock prices modeled by Brownian motion. The exact solution for determining Indonesian option prices is obtained by a formula in [2]. Whereas, the numerical solution is obtained by the implicit finite difference method (12) with $\alpha=0, \beta=1$ and $H=\frac{1}{2}$.

Moreover, if we set $\alpha=1$ and $\beta=0$ in (12), then we get a similar trend of option prices as shown in Figure 4. As can be seen, both solutions overlap each other. In other words, the numerical solution is similar to the analytical solution.

In Examples 1, 2, 3 and 4, we choose small $\Delta S$ and $\Delta \tau$ values. The implicit finite difference scheme can still produce Indonesian option prices using these values. In other words, even though the values chosen are very small, it still produces option prices. We need to mention here that the calculation process takes a longer time. In addition, we can see that trends
Table 1. Convergence results of the scheme (12)

\begin{tabular}{|c|c|c|c|c|}
\hline$\Delta S$ & $\Delta \tau$ & Value & Difference & Ratio \\
\hline 10.00000 & 0.001000000 & 30.7251 & & \\
\hline 5.00000 & 0.000500000 & 30.8103 & 0.0852 & \\
\hline 2.50000 & 0.000250000 & 30.8352 & 0.0249 & 3.4217 \\
\hline 1.25000 & 0.000125000 & 30.8433 & 0.0081 & 3.0741 \\
\hline 0.62500 & 0.000062500 & 30.8463 & 0.0030 & 2.7000 \\
\hline 0.31250 & 0.000031250 & 30.8475 & 0.0012 & 2.5000 \\
\hline 0.15625 & 0.000015625 & 30.8480 & 0.0005 & 2.4000 \\
\hline
\end{tabular}

and visible shapes of option price solutions of the proposed scheme are similar to the option price solutions in [2] (Example 5). Therefore, it can be concluded that the implicit finite difference scheme used to determine Indonesian option prices is stable and convergent.

\section{Conclusions}

In this paper, we apply an implicit finite difference method to solve Indonesian option pricing problems. Given that Jakarta Composite Index is long-range dependent, an MFBM is used to model the stock returns. The implicit finite difference scheme has been developed to solve a partial differential equation that is used to determine Indonesian option prices. We study the stability and convergence of the implicit finite difference scheme for Indonesian option pricing. We also present several examples of numerical solutions for Indonesian option pricing. Based on theoretical analysis and numerical solutions, the scheme proposed in this paper is efficient and reliable.

\section{Acknowledgements}

The authors gratefully acknowledge that this research was supported by Universitas Sanata Dharma and Universitas Gadjah Mada. The authors also thank the referees for their comments and suggestions which improve the paper significantly.

\section{Appendix}

\section{A Review of a mixed fractional Brownian mo- tion}

In Appendix A, we recall several definitions and lemma which are used in this paper.

Definition A.1. [21] Let $H \in(0,1)$ be given. A fractional Brownian motion $B^{H}=\left(B_{t}^{H}\right)_{t \geq 0}$ of Hurst index $H$ is a continuous and centered Gaussian process with covariance function

$$
E\left[B_{t}^{H}, B_{u}^{H}\right]=\frac{1}{2}\left(|t|^{2 H}+|u|^{2 H}-|t-u|^{2 H}\right),
$$

for all $t, u>0$.

A FBM is a generalization of the standard Brownian motion. To see this take $H=\frac{1}{2}$ in the Definition A.1. Standard Brownian motion has been employed to model stock prices in 
the Black-Scholes model. However, it cannot model time series with long-range dependence (long memory). It is known that a FBM is able to model time series with long-range dependence for $\frac{1}{2}<H<1$.

One main problem of using a FBM in financial models is that it exhibits arbitrage which is usually excluded in the modeling. To avoid the possibility of arbitrage, Cheridito [22] introduced an MFBM.

Definition A.2. $[22,23]$ A mixed fractional Brownian motion of parameters $\alpha, \beta$ and $H$ is a process $M^{H}=\left(M_{t}^{H, \alpha, \beta}\right)_{t \geq 0}$, defined on a probability space $\left(\Omega, \mathcal{F}, \mathbb{P}^{H}\right)$ by

$$
M_{t}^{H, \alpha, \beta}=\alpha B_{t}+\beta B_{t}^{H}, \quad t \geq 0,
$$

where $\left(B_{t}\right)_{t \geq 0}$ is a Brownian motion and $\left(B_{t}^{H}\right)_{t \geq 0}$ is an independent FBM of Hurst index $H$.

We rewrite the following lemma which is derived from the Ito formula [21, 24] and properties of an MFBM. The lemma will be used later in option pricing based on stock price modeled by an MFBM.

Lemma A.3. [25] Let $f=f\left(t, S_{t}\right)$ is a differentiable function. Let $\left(S_{t}\right)_{t \geq 0}$ be a stochastic process given by

$$
d S_{t}=\mu S_{t} d t+\sigma_{1} S_{t} d B_{t}+\sigma_{2} S_{t} d B_{t}^{H},
$$

where $B_{t}$ is a Brownian motion, $B_{t}^{H}$ is a FBM, and assume that $B_{t}$ and $B_{t}^{H}$ are independent, then we have

$$
\begin{aligned}
d f= & \left(\frac{\partial f}{\partial t}+\mu S_{t} \frac{\partial f}{\partial S_{t}}+\frac{\sigma_{1}^{2} S_{t}^{2}}{2} \frac{\partial^{2} f}{\partial S_{t}^{2}}+H \sigma_{2}^{2} S_{t}^{2} t^{2 H-1} \frac{\partial^{2} f}{\partial S_{t}^{2}}\right) d t \\
& +\sigma_{1} S_{t} \frac{\partial f}{\partial S_{t}} d B_{t}+\sigma_{2} S_{t} \frac{\partial f}{\partial S_{t}} d B_{t}^{H}
\end{aligned}
$$

\section{B Proofs}

\section{Proof of Lemma 1}

Proof. Using Lemma A.3 with $\mu=r, \sigma_{1}=\alpha \sigma$ and $\sigma_{2}=\beta \sigma$ and taking $f\left(S_{t}\right)=\ln \left(S_{t}\right)$, be obtained:

$$
\begin{aligned}
d \ln \left(S_{t}\right) & =\left(r-\frac{1}{2}(\alpha \sigma)^{2}-(\beta \sigma)^{2} H t^{2 H-1}\right) d t \\
& +\alpha \sigma d B_{t}+\beta \sigma d B_{t}^{H}
\end{aligned}
$$

and hence,

$$
\ln \left(\frac{S_{t}}{S_{0}}\right)=r t-\frac{1}{2}(\alpha \sigma)^{2} t-\frac{1}{2}(\beta \sigma)^{2} t^{2 H}+\alpha \sigma B_{t}+\beta \sigma B_{t}^{H},
$$

which can be related as (2).

\section{Proof of Theorem 2}

Proof. To prove the statement, a portfolio consisting an option $V(t, S)$ and a quantity $q$ of stock, will be first set, i.e.

$$
\Pi=V(t, S)-q S .
$$

Thus, changes in portfolio value in a short time can be written as

$$
d \Pi=d V(t, S)-q d S .
$$

Now, applying Lemma A.3 and $f\left(t, S_{t}\right)=V(t, S)$, we obtain

$$
\begin{aligned}
d V= & \left(\frac{\partial V}{\partial t}+r S \frac{\partial V}{\partial S}+\frac{1}{2}(\alpha \sigma S)^{2} \frac{\partial^{2} V}{\partial S^{2}}+(\beta \sigma S)^{2} H t^{2 H-1} \frac{\partial^{2} V}{\partial S^{2}}\right) d t \\
& +\alpha \sigma S \frac{\partial V}{\partial S} d B_{t}+\beta \sigma S \frac{\partial V}{\partial S} d B_{t}^{H}
\end{aligned}
$$

Substituting (B.3) and (1) into (B.2), we have

$$
\begin{aligned}
d \Pi= & \left(\frac{\partial V}{\partial t}+r S\left(\frac{\partial V}{\partial S}-q\right)+\frac{1}{2}(\alpha \sigma S)^{2} \frac{\partial^{2} V}{\partial S^{2}}\right. \\
& \left.+(\beta \sigma S)^{2} H t^{2 H-1} \frac{\partial^{2} V}{\partial S^{2}}\right) d t+\alpha \sigma S\left(\frac{\partial V}{\partial S}-q\right) d B_{t} \\
& +\beta \sigma S\left(\frac{\partial V}{\partial S}-q\right) d B_{t}^{H} .
\end{aligned}
$$

Further, we choose $q=\frac{\partial V}{\partial S}$ to eliminate the random noise. Then we get

$$
d \Pi=\left(\frac{\partial V}{\partial t}+\frac{1}{2}(\alpha \sigma S)^{2} \frac{\partial^{2} V}{\partial S^{2}}+(\beta \sigma S)^{2} H t^{2 H-1} \frac{\partial^{2} V}{\partial S^{2}}\right) d t .
$$

On the other hand, the portfolio becomes riskless if the portfolio yield is only determined by the risk-free interest rate $r$, which satisfies $d \Pi=r \Pi d t$. From (B.1), we have

$$
r \Pi d t=r(V-q S) d t=\left(r V-r S \frac{\partial V}{\partial S}\right) d t,
$$

and also from (B.4) and (B.5), we get

$$
\begin{gathered}
\left(\frac{\partial V}{\partial t}+\frac{1}{2}(\alpha \sigma S)^{2} \frac{\partial^{2} V}{\partial S^{2}}+(\beta \sigma S)^{2} H t^{2 H-1} \frac{\partial^{2} V}{\partial S^{2}}\right) d t \\
=\left(r V-r S \frac{\partial V}{\partial S}\right) d t
\end{gathered}
$$

which yields (3).

\section{Proof of Proposition 3}

Proof. Since $\left|\vartheta_{j}\right| \geq 1$ and using (22) for $k=1$, we have

$$
\left|\xi^{1}\right|=\frac{1}{\left|\vartheta_{j}\right|}\left|\xi^{0}\right| \leq\left|\xi^{0}\right|
$$

If $\left|\xi^{k-1}\right| \leq\left|\xi^{0}\right|$, then using (22), we obtain

$$
\left|\xi^{k}\right|=\frac{1}{\left|\vartheta_{j}\right|}\left|\xi^{k-1}\right| \leq \frac{1}{\left|\vartheta_{j}\right|}\left|\xi^{0}\right| \leq\left|\xi^{0}\right| \text {. }
$$

This completes the proof.

\section{Proof of Theorem 4}

Proof. Using Proposition 3 and (19), we obtain

$$
\left\|\varepsilon^{k}\right\|_{2} \leq\left\|\varepsilon^{0}\right\|_{2}, \quad k=1,2, \ldots, N,
$$

which means that the difference scheme (12) is unconditionally stable.

\section{Proof of Proposition 5}

Proof. From (26) and (30), we have

$$
\begin{aligned}
\left\|\boldsymbol{R}^{k}\right\|_{2} & \leq\left(\sum_{j=1}^{M-1} C_{1}\left(\Delta \tau+(\Delta S)^{2}\right)^{2} \Delta S\right)^{\frac{1}{2}} \\
& \leq C_{1}\left(\Delta \tau+(\Delta S)^{2}\right) \sqrt{M \Delta S} \\
& \leq C_{1} \sqrt{S_{\max }}\left(\Delta \tau+(\Delta S)^{2}\right)
\end{aligned}
$$


where $k=1,2, \ldots, N$. If the series of the right hand side of (32) convergent, then there is a positive constant $C_{2}^{k}$, such that

$$
\left|\rho^{k}\right| \equiv\left|\rho^{k}(l)\right| \leq C_{2}^{k}\left|\rho^{1}\right| \equiv C_{2}^{k}\left|\rho^{1}(l)\right|
$$

Then, we have

$$
\left|\rho^{k}\right| \leq C_{2}\left|\rho^{1}\right|,
$$

where $C_{2}=\max \left\{C_{2}^{k} \mid k=1,2, \ldots, N\right\}$. By using (29) and (31), we have $\varrho^{0}=0$. For $k=1$, from (38) and (B.7), we get

$$
\left|\varrho^{1}\right|=\Delta \tau\left|\rho^{1}\right| \leq C_{2} \Delta \tau\left|\rho^{1}\right|
$$

Suppose now that $\left|\varrho^{n}\right| \leq C_{2} n \Delta \tau\left|\rho^{1}\right|, n=1,2, \ldots, k-1$, then by using 38 and B.7, we obtain

$$
\begin{aligned}
\left|\varrho^{k}\right| & \leq \frac{1}{\left|\vartheta_{j}\right|} C_{2}(k-1) \Delta \tau\left|\rho^{1}\right|+\frac{1}{\left|\vartheta_{j}\right|} C_{2} \Delta \tau\left|\rho^{1}\right| \\
& \leq\left(\frac{(k-1)}{k\left|\vartheta_{j}\right|}+\frac{1}{k\left|\vartheta_{j}\right|}\right) C_{2} k \Delta \tau\left|\rho^{1}\right| \\
& \leq C_{2} k \Delta \tau\left|\rho^{1}\right|
\end{aligned}
$$

This completes the proof.

\section{Proof of Theorem 6}

Proof. By using Proposition and (31), (32) and (B.6), we obtain

$$
\begin{aligned}
\left\|\boldsymbol{\epsilon}^{k}\right\|_{2} & \leq C_{2} k \Delta \tau\left\|\boldsymbol{R}^{1}\right\|_{2} \\
& \leq C_{1} C_{2} k \Delta \tau \sqrt{S_{\max }}\left(\Delta \tau+(\Delta S)^{2}\right)
\end{aligned}
$$

Because $k \Delta \tau \leq T$, we have

$$
\left\|\boldsymbol{\epsilon}^{k}\right\|_{2} \leq C\left(\Delta \tau+(\Delta S)^{2}\right)
$$

where $C=C_{1} C_{2} T \sqrt{S_{\max }}$

\section{REFERENCES}

[1] Keputusan Direksi PT Bursa Efek Jakarta Nomor Kep310/BEJ/09-2004 tentang peraturan nomor II-D tentang perdagangan opsi saham, Online available from https://idx.co.id/media/1344/3.pdf. [Accessed: 22-Jan-2020].

[2] Gunardi, J.H.M. Anderluh, J.A.M. Van Der Weide, Subanar, and S. Haryatmi, Indonesian options, Rep. 06-11, Delft Univ. Technol. Netherlands, 2006.

[3] Gunardi, J.A.M. Van Der Weide, Subanar, and S. Haryatmi, P(I)DE approach for Indonesian options pricing, Journal of the Indonesian Mathematical Society, vol. 14, no. 1, p. 37-45, 2008.

[4] Gunardi, The greeks of indonesian call option, Far East Journal of Mathematical Sciences, vol. 101, no. 10, pp. 2111-2120, 2017.
[5] D.O. Cajueiro and B.M. Tabak, Testing for long-range dependence in world stock markets, Chaos, Solitons and Fractals, vol. 37, no. 3, pp. 918-927, 2008.

[6] C. Necula and A.N. Radu, Long memory in Eastern European financial markets returns, Economic Research - Ekonomska Istraživanja, vol. 25, no. 2, pp. 316-377, Jan. 2012.

[7] D. Fakhriyana, Irhamah, and K. Fithriasari, Modeling Jakarta composite index with long memory and asymmetric volatility approach, AIP Conference Proceedings, vol. 2194, no. 020025, 2019.

[8] Y. Hu and B. Øksendal, Fractional white noise calculus and applications to finance, Infinite Dimensional Analysis, Quantum Probability and Related Topics, vol. 06, no. 01, pp. $1-32,2003$.

[9] P. Cheridito, Arbitrage in fractional Brownian motion models, Finance and Stochastics, vol. 7, no. 4, pp. 533-553, 2003.

[10] C.E. Murwaningtyas, S.H. Kartiko, Gunardi, and H.P. Suryawan, European option pricing by using a mixed fractional Brownian motion, Journal of Physics: Conference Series, vol. 1097, no. 1, 2018.

[11] C.E. Murwaningtyas, S.H. Kartiko, Gunardi, and H.P. Suryawan, Option pricing by using a mixed fractional Brownian motion with jumps, Journal of Physics: Conference Series, vol. 1180, no. 1, p. 012081, Sep. 2019.

[12] W. Chen, B. Yan, G. Lian, and Y. Zhang, Numerically pricing American options under the generalized mixed fractional Brownian motion model, Physica A, vol. 451, pp. 180-189, 2016.

[13] B.L.S. Prakasa Rao, Pricing geometric Asian power options under mixed fractional Brownian motion environment, Physica A, vol. 446, pp. 92-99, 2016.

[14] W.G.G. Zhang, Z. Li, and Y.J.J. Liu, Analytical pricing of geometric Asian power options on an underlying driven by a mixed fractional Brownian motion, Physica A, vol. 490, pp. 402-418, 2018

[15] L. Wang, R. Zhang, L. Yang, Y. Su, and F. Ma, Pricing geometric Asian rainbow options under fractional Brownian motion, Physica A, vol. 494, pp. 8-16, 2018.

[16] L. Sun, Pricing currency options in the mixed fractional Brownian motion, Physica A, vol. 392, no. 16, pp. 3441-3458, 2013. 
[17] K.H. Kim, S. Yun, N.U. Kim, and J.H. Ri, Pricing formula for European currency option and exchange option in a generalized jump mixed fractional Brownian motion with time-varying coefficients, Physica A, vol. 522, pp. 215-231, 2019.

[18] K.H. Kim, N.U. Kim, D.C. Ju, and J.H. Ri, "Efficient hedging currency options in fractional Brownian motion model with jumps,” Physica A, vol. 539, p. 122868, 2020.

[19] L.V. Ballestra, G. Pacelli, and D. Radi, A very efficient approach for pricing barrier options on an underlying described by the mixed fractional Brownian motion, Chaos, Solitons and Fractals, vol. 87, pp. 240-248, 2016.

[20] L. Song and W. Wang, Solution of the fractional Black-Scholes option pricing model by finite difference method, Abstract and Applied Analysis, vol. 2013, 2013.

[21] F. Biagini, Y. Hu, B. Øksendal, and T. Zhang, Stochastic Calculus for Fractional Brownian Motion and Applications.
Springer, 2008.

[22] P. Cheridito, Mixed fractional Brownian motion, Bernoulli, vol. 7, no. 6, pp. 913-934, 2001.

[23] M. Zili, On the mixed fractional Brownian motion, On the mixed fractional Brownian motion, vol. 2006, pp. 1-9, 2006.

[24] T.E. Duncan, Y. Hu, and B. Pasik-Duncan, Stochastic calculus for fractional Brownian motion I. Theory, SIAM Journal on Control and Optimization, vol. 38, no. 2, pp. 582-612, 2000.

[25] Z. Yang, Efficient valuation and exercise boundary of American fractional lookback option in a mixed jump-diffusion model, International Journal of Financial Engineering, vol. 04, no. 02n03, p. $1750033,2017$. 